\title{
Dispersion of a passive scalar within and above an urban street network
}

Article

Accepted Version

Goulart, E. V., Coceal, O. and Belcher, S. E. (2018) Dispersion of a passive scalar within and above an urban street network. Boundary-Layer Meteorology, 166 (3). pp. 351-366. ISSN 0006-8314 doi: https://doi.org/10.1007/s10546-017-0315-5 Available at https://centaur.reading.ac.uk/72996/

It is advisable to refer to the publisher's version if you intend to cite from the work. See Guidance on citing.

To link to this article DOI: http://dx.doi.org/10.1007/s10546-017-0315-5

Publisher: Springer

All outputs in CentAUR are protected by Intellectual Property Rights law, including copyright law. Copyright and IPR is retained by the creators or other copyright holders. Terms and conditions for use of this material are defined in the End User Agreement.

\section{www.reading.ac.uk/centaur}

\section{CentAUR}

Central Archive at the University of Reading

Reading's research outputs online 


\title{
1. Dispersion of a passive scalar within and above an urban street network
}

\author{
3 EV Goulart · O Coceal · SE Belcher
}

5 Received: DD Month YEAR / Accepted: DD Month YEAR

6 Abstract The transport of a passive scalar from a continuous point-source release in an urban street network is studied using direct numerical simulation (DNS). Dispersion through the network is characterized by evaluating horizontal fluxes of scalar within and above the urban canopy and vertical exchange fluxes through the canopy top. The relative magnitude and balance of these fluxes are used to distinguish three different regions relative to the source location: a near-field region, a transition region and a far-field region. The partitioning of each of these fluxes into mean and turbulent parts is computed. It is shown that within the canopy the horizontal turbulent flux in the street network is small, whereas above the canopy it comprises a significant fraction of the total flux. Vertical fluxes through the array top are predominantly turbulent. The mean and turbulent fluxes are respectively parametrized in terms of an advection velocity and a detrainment velocity and the parametrization incorporated into a simple box-network model. The model treats the coupled dispersion problem within and above the street network in a unified way and predictions of mean concentrations compare well with the DNS data. This demonstrates the usefulness of the box-network approach for process studies and interpretation of results from more detailed numerical simulations.

EV Goulart

Department of Meteorology, University of Reading, PO Box 243, Reading, RG6 6BB, UK

Tel.: +55-27-40092177

Fax: +55-27-40092148

E-mail: elisa.goulart@ufes.br

Present address:Federal University of Espirito Santo, Vitoria, Brazil

O Coceal

National Centre for Atmospheric Science (NCAS), Department of Meteorology, University of Reading, PO Box 243, Reading, RG6 6BB, UK

SE Belcher

Department of Meteorology, University of Reading, PO Box 243, Reading, RG6 6BB, UK 
${ }_{24}$ Keywords Dispersion model $\cdot$ Street network $\cdot$ Urban dispersion

\section{Introduction}

Noteworthy studies of dispersion in urban areas include a number of detailed field and scaled model experiments (e.g., Davidson et al., 1995, 1996; Macdonald et al., 1997, 1998; Yee and Biltoft, 2004; Yee et al., 2006; Hilderman et al., 2007; Carpentieri et al., 2009) as well as high-resolution numerical simulations (e.g., Hanna et al., 2002; Milliez and Carissimo, 2007; Branford et al., 2011; Philips et al., 2013). These have provided insight into how the presence of buildings modifies concentration levels in urban areas and what flow and dispersion processes contribute to these differences. For a general context, we refer the reader to reviews included in Britter and Hanna (2003) and Branford et al. (2011). Against the apparent complexity of empirical results, it is helpful to ask whether a core set of robust dispersion processes can be identified that could be of practical use in building approaches to model dispersion in the urban environment.

The need for urban dispersion models suitable for operational air-quality and emergency-response applications in particular requires novel approaches that can represent potentially complex turbulent flow processes in a simplified way. Recently Belcher et al. (2015) have proposed a simple approach for modelling dispersion in a street network regime, where the buildings are close enough that a distinct network of streets emerges. The methodology follows Soulhac (2000) who developed the governing equations for a family of network models, together with methods for estimating the model parameters, which then led to the development of an operational dispersion model, SIRANE (Soulhac et al., 2011, 2012, 2016). Hamlyn et al. (2007) constructed a much simpler network model for dispersion through an array of cubes, showing impressive agreement with measurements made in a water channel by Hilderman et al. (2007). Belcher (2005) and Belcher et al. (2015) developed an analytical model for the dispersion of a passive scalar within a regular street network, which showed that the concentration is given in a closed form solution that includes an explicit dependence on the basic geometrical and flow parameters, which combine into only three effective parameters. Despite the important theoretical insight that this solution provides, the authors found that the solution is restricted to the so-called near-field regime, where the vertical dispersion is dominated by detrainment out of the street network into the flow above. Beyond the near-field region the re-entrainment of material back into the street network needs to be taken into account; this cannot be handled analytically in a robust way, although Belcher et al. (2015) gained additional insight through the use of a toy model for re-entrainment. Moreover, the dispersion above the canopy must be modelled too, as the interaction between the canopy and the flow above is a two-way process.

Against this background, our study is motivated by the aim of developing a simple model based on a minimal set of processes that will produce reli- 
able estimates of the mean concentration both in the near-field region and beyond. The requirement of simplicity stems from the need for viable approaches for modelling in emergency-response or regulatory contexts. The interest in a process-based approach ensures that the very design of the model rests upon sound physical insights. This requires that we have an understanding of which processes are the most important to include and how best to parametrize them. The objectives are therefore two-fold: (i) In order to better understand the dispersion processes both within and above the urban canopy and how they interact we propose to analyze data from a previously-performed direct numerical simulation (DNS) over an array of cubical buildings (Branford et al., 2011). (ii) We extend the model of Belcher et al. (2015) to treat the dispersion both within and above the street network in a coupled way; this then explicitly represents the re-entrainment of material into the street network beyond the near-field regime.

The paper is structured as follows: Sect. 2 outlines the DNS dataset and method of analysis adopted; Sect. 3 is devoted to reporting and discussing results from analysis of the DNS data on the horizontal and vertical transport within and above the street network. In Sect. 4 the main results are used to formulate a simple street network model and to perform numerical experiments and parameter sensitivity studies with it. Conclusions are given in Sect. 5.

\section{Numerical data and analysis}

This section briefly outlines the DNS dataset and the method of analyzing the data.

\subsection{Direct numerical simulations over a regular array}

DNS data of Branford et al. (2011) are used here. The DNS models the dispersion of passive scalars by numerically solving the scalar equation,

$$
\frac{\partial c}{\partial t}+\mathbf{u} \cdot \nabla c=D \nabla^{2} c+S,
$$

where $c$ is the concentration of scalar, $\mathbf{u}$ is the instantaneous velocity field vector, $D$ is the molecular diffusivity and $S$ is a source term. The instantaneous turbulent velocity field $\mathbf{u}$ is a solution of the Navier-Stokes equations. The Schmidt number $S c \equiv \nu / D=1$ in all the simulations. A steady point-source release near the ground was simulated, so that the source term is given by $S=q \delta^{3}\left(\mathbf{x}-\mathbf{x}_{\mathbf{s}}\right)$, where $q$ is a constant source emission rate, $\mathbf{x}$ is the position vector, $\mathbf{x}_{\mathbf{s}}$ is the position vector of the source and $\delta^{3}(\mathbf{x})$ is the Dirac delta function. In practice, the source is discretized as a Gaussian ball over a few grid points. The computational set-up, consisting of a regular array of cubes, allowed for multiple independent scalar fields to be modelled during each simulation. Figure 1a shows the computational domain and source locations, with 


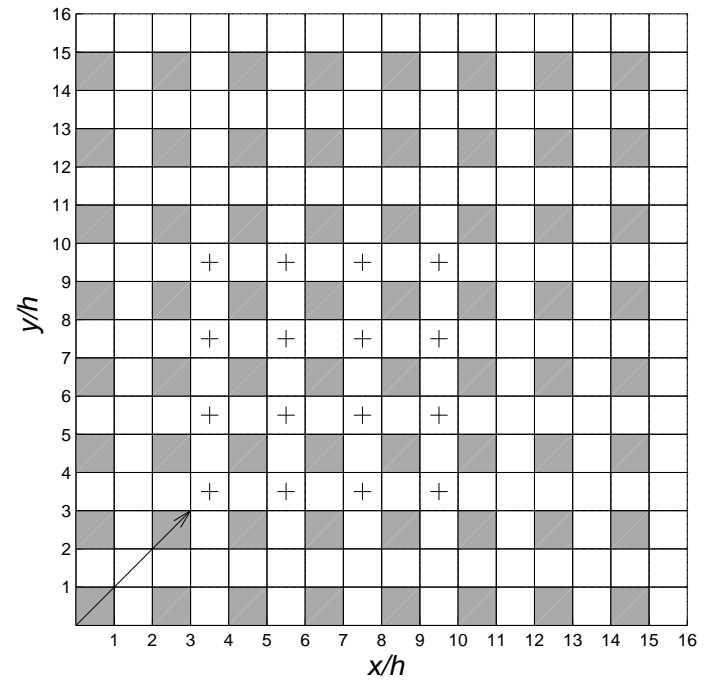

(a)

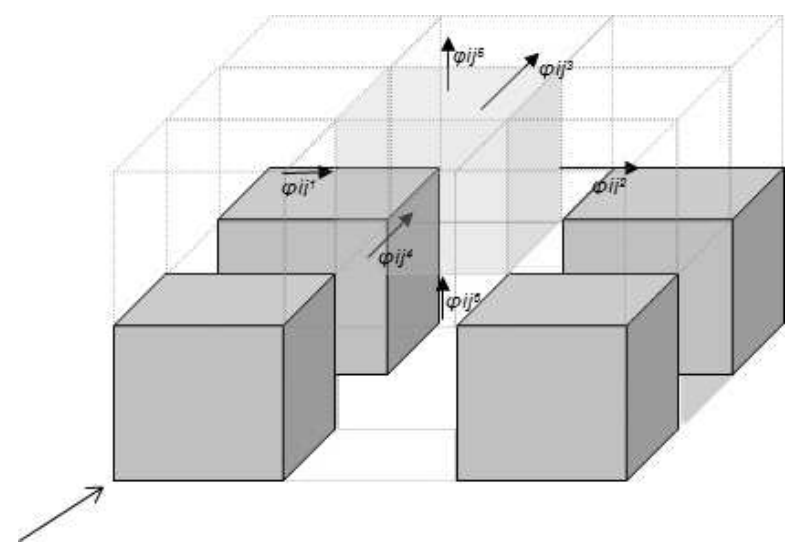

(b)

Fig. 1 (a) Plan view of the computational domain in the DNS. Plus signs denote locations of the ground sources. (b) Schematic of fluxes through a box above an intersection.

a mean flow direction of $45^{\circ}$ as indicated in the figure. We note that the flow is symmetric with respect to the two horizontal components, $u$ and $v$. Much existing work in the literature has dealt with cases where the mean flow is either aligned with or perpendicular to streets. However, these idealised cases almost never occur under actual meteorological conditions; indeed they give rise to somewhat artificial flow regimes. A mean flow oblique to the streets constitutes a more realistic scenario.

The DNS employed dimensionless units, with lengths normalised by the building height $h$, velocities normalised by the friction velocity $u_{\tau}$ and with the 
density of air $\rho=1$. All quantities and parameters are given in corresponding dimensionless units unless otherwise stated.

Time- and ensemble- averaged concentration statistics showed very good agreement with experimental data (Branford et al., 2011). The data generated from these simulations are here analyzed within a box-network framework, described in the next section.

\subsection{Analysis within a box-network framework}

In the box-network framework an array of buildings is considered as forming a network of 'streets' (here defined as the space between adjacent buildings) joined at 'intersections'; each of the streets and intersections can be thought of as a box, through whose facets a scalar can enter or leave. Goulart et al. (2016) showed that to a first approximation the scalar is generally well mixed in each such box except near the source and the edges of the plume. Further layers of boxes can be envisaged above the streets, intersections and buildings as shown in Fig. 1b. The transport of scalars in such a street network can be analyzed by considering the fluxes entering and leaving the boxes. Such an approach forms the basis of a family of street network dispersion models (Soulhac, 2000; Belcher, 2005; Hamlyn et al., 2007; Soulhac et al., 2011; Belcher et al., 2015), a version of which will be presented in Sect. 4. To inform the development of such a model, in Sect. 3 scalar fluxes over the facets of the boxes are computed from the DNS data.

\section{Scalar transport through a street network: results from DNS}

Dispersion of scalars through the street network is controlled by horizontal fluxes within and above the urban canopy and by vertical exchange fluxes through the canopy top linking these two regions. Each of these fluxes can be formally decomposed into a mean and a turbulent component,

$$
\left\langle\overline{c u_{i}}\right\rangle=\left\langle\bar{c} \overline{u_{i}}\right\rangle+\left\langle\overline{c^{\prime} u_{i}^{\prime}}\right\rangle,
$$

where $c$ is the instantaneous concentration and $u_{i}$ is an instantaneous velocity component perpendicular to the relevant facet. In Eq. 2 the overbar denotes time-averaging and angled brackets denote spatial averaging over a facet. Horizontal and vertical fluxes within and above the array and their mean and turbulent components are computed from the DNS data. The results are then applied in the configuration of the network model in Sect. 4.

\subsection{Horizontal scalar fluxes within and above the canopy}

Horizontal scalar fluxes within the canopy calculated from the DNS data are plotted as a fraction of the total flux at different locations from the source in 


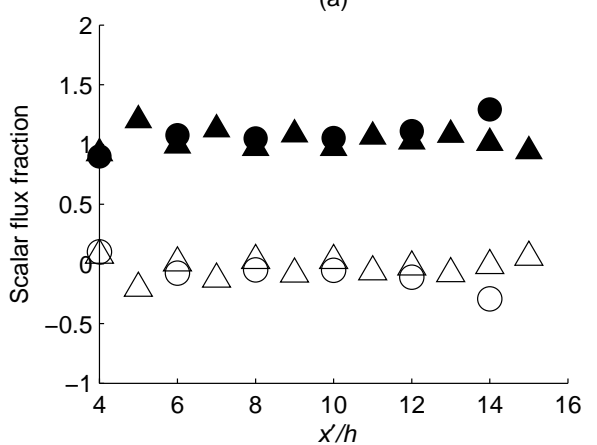

(b)

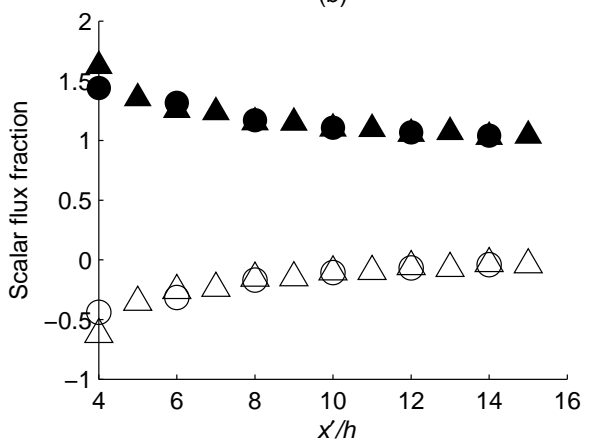

(c)

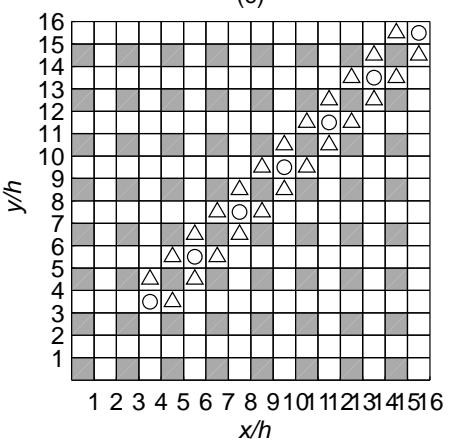

Fig. 2 Ratio of horizontal scalar fluxes, (a) within the canopy, (b) above the canopy. Filled symbols: ratio of mean to total flux $\langle\bar{c} \bar{u}\rangle /\langle\overline{c u}\rangle$. Empty symbols: ratio of turbulent to total flux $\left\langle\overline{c^{\prime} u^{\prime}}\right\rangle /\langle\overline{c u}\rangle$. (c) Sampling locations. Circles: intersections. Triangles: streets. $x \prime$ represents the spanwise direction.

Fig. 2a. The locations of the boxes in which the fluxes were calculated lie along three transects, as shown in Fig. 2c. We note that the middle transect involves only intersections and the other two transects involve only streets. The results for the latter two transects have been averaged. It is apparent from Fig. 2a that, for both streets and intersections, the mean flux is much larger than the turbulent flux irrespective of distance from the source. The average value of the ratio of mean to total vertical flux in the canopy is $\langle\bar{c} \bar{u}\rangle /\langle\overline{c u}\rangle=0.99$ (and similarly for the $v$ components, by symmetry). This ratio is relatively constant throughout the array.

Figure $2 \mathrm{~b}$ shows flux fractions along corresponding transects for the layer of boxes just above the buildings. The most noteworthy difference is that the turbulent fluxes are now negative and comprise a significant fraction of the total flux (up to 0.5). The mean flux fraction is always larger than 1, with a maximum value of about 1.5. The average value of the ratio of mean to total vertical flux just above the canopy is $\langle\bar{c} \bar{u}\rangle /\langle\overline{c u}\rangle=1.27$. The corresponding average turbulent flux ratio is therefore $\left\langle\overline{c^{\prime} u^{\prime}}\right\rangle /\langle\overline{c u}\rangle=-0.27$. The occurrence 
of this large counter-gradient turbulent flux ratio above the canopy contrasts with the small positive value of 0.01 within the canopy. The origin of these negative turbulent fluxes is unclear; a possible mechanism could involve ejections associated with coherent structures above the canopy (e.g., Coceal et al., 2007).

\subsection{Vertical scalar fluxes through the canopy top}

The mean and turbulent components of the vertical flux through the top of the array are shown in Fig. 3. The mean vertical flux $\langle\bar{c} \bar{w}\rangle$ is always positive in the intersections and always negative in the streets (Fig. 3a). Since $\bar{c}$ is always positive, the sign of $\langle\bar{c} \bar{w}\rangle$ is determined by that of $\bar{w}$. Hence, the pattern of mean inflow or outflow is determined by the mean vertical velocity pattern. The vertical velocity averaged over the top facet of a street $\langle\bar{w}\rangle$ is indeed downward, whereas it is upward over an intersection (not shown). We note that the mean vertical flux from the first intersection (which contains the source) is anomalously low; see below.

There is little difference between the turbulent fluxes $\left\langle\overline{c^{\prime} w^{\prime}}\right\rangle$ for streets and intersections (Fig. 3b). They are positive for both streets and intersections in the near-field region, but becomes slightly negative from the third intersection onwards. The maximum turbulent flux is about an order of magnitude larger than the maximum mean flux. The turbulent flux decays much quicker with distance from the source than the mean vertical flux. This may be because turbulent scalar exchanges take place in both directions, and hence tend to equalise quicker.

The ratios of the mean and turbulent vertical fluxes to the total vertical flux (Fig. 3c) reveal the following: (i) Up to a distance of about four building heights from the source the turbulent flux is the dominant component for both streets and intersections. (ii) However, far from the source (beyond a distance of about ten building heights) there is considerable scatter in the flux ratio. This is because both the turbulent and mean fluxes are small in the far-field region. The turbulent flux is slightly negative for intersections and both turbulent and mean flux are negative for streets.

\subsection{Horizontal vs. vertical transport}

The vertical flux through the canopy top exerts a strong control on how a plume spreads through a street network. Vertical detrainment from the canopy results in a reduction in the amount of material available to disperse horizontally through the canopy; this should cause a rapid fall-off of the concentration with distance from the source. However, material can also be re-entrained into the canopy further downstream. The balance between detrainment and reentrainment is not the only factor that determines the subsequent horizontal fall-off. Equally important is the lateral spread through the canopy. 

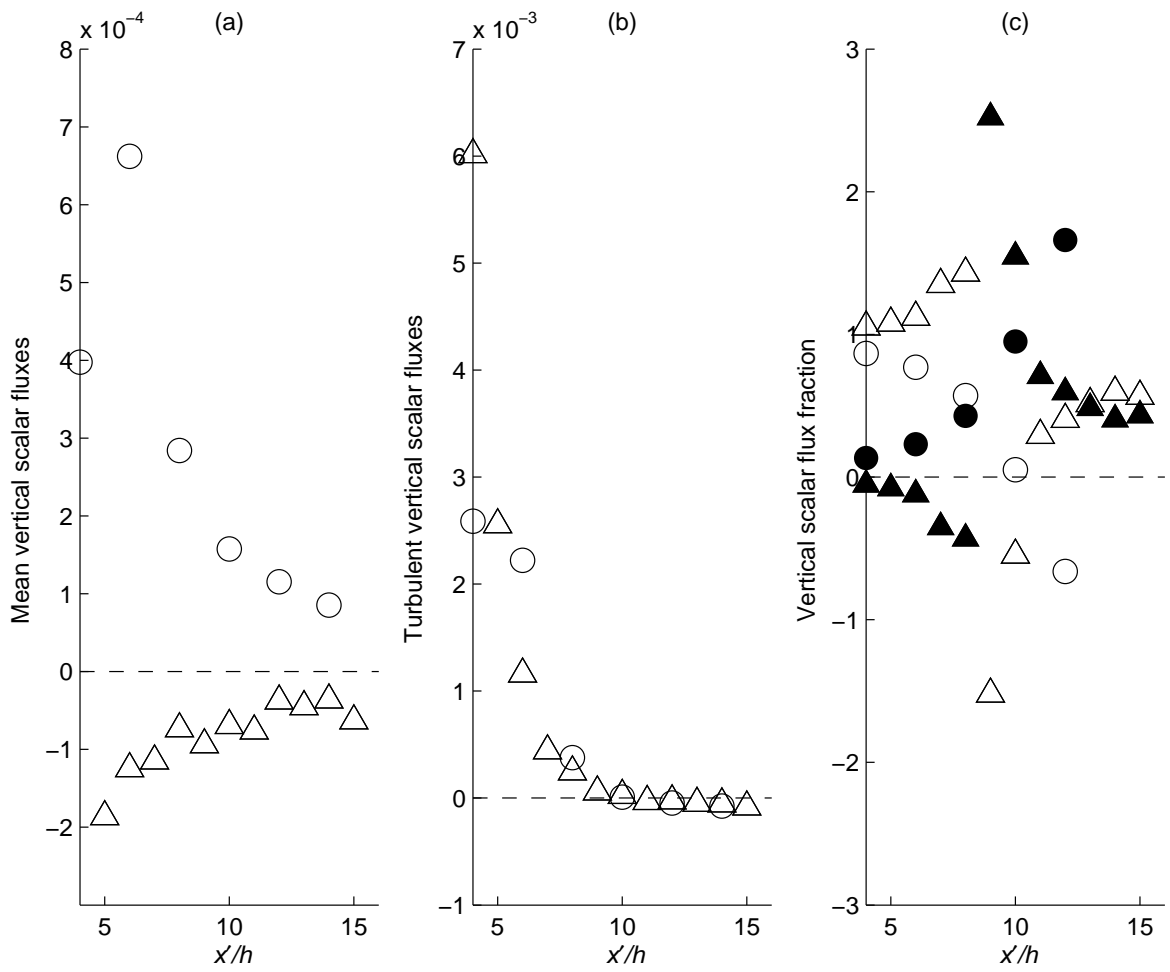

Fig. 3 Vertical fluxes through the canopy top at the same sampling locations as in 2c. (a) mean, (b)turbulent, (c) ratio of mean (filled symbols) and turbulent (empty symbols) to total. Circles: intersections. Triangles: streets. Fluxes have been normalized using the release rate $q$.

Figures $4 \mathrm{a}, 4 \mathrm{~b}$ and $4 \mathrm{c}$ respectively show the horizontal scalar flux through the canopy, vertical flux through the canopy top, and the ratio of vertical to horizontal flux, as a function of distance from the source. There is a rapid decrease in both the horizontal and vertical fluxes up to the third intersection downstream, followed by a much more gradual decrease thereafter. The total horizontal flux behaves in roughly the same way in streets and intersections. In contrast, there is a clear difference between the vertical fluxes in the streets and the intersections; the near-field and far-field behaviours also differ. The vertical flux in the intersections is generally positive, so that material is nearly always detrained out of the intersections into the air above. The vertical flux in the streets is positive close to the source but changes sign between the second and third intersections downstream. This implies that re-entrainment begins to exceed detrainment very rapidly downstream of the release, at least in the present set-up. The magnitude of the flux in the intersections is larger than that from the streets in the near-field region (up to the third intersection downstream of the release). As noted earlier, the vertical flux in the first intersection (which contains the source) is anomalously low compared to that 
in the streets immediately adjacent to it. This arises because material released in an intersection is rapidly swept to the next streets downstream, caught in the wakes of adjacent buildings and pushed upwards by a strong updraft (Coceal et al., 2014). This gives rise to 'secondary wake sources' (Vincent, 1978) in the relevant streets, which detrain material at a much higher rate than in the intersection where the source is located. Secondary sources were also observed in previous experimental studies, e.g. Davidson et al. $(1995,1996)$.

Fig. 4c shows that the magnitude of the vertical flux is generally less than a quarter of the horizontal flux, except at the location furthest from the source (where both fluxes are small). After an initial increase with distance from the source location this ratio decreases steadily up to the third intersection. Beyond this point there is a difference in the behaviour in intersections and streets. In intersections there is a continual slow decrease towards zero. In streets the ratio becomes negative because the vertical flux changes sign due to re-entrainment into the canopy.

It is instructive to decompose the vertical flux into an upward component (detrained flux) and a downward component (entrained flux). Figure 4d shows the ratio of the downward flux to the upward flux for the same intersections and streets. The downward flux is a small fraction (around 0.05) of the upward flux in the first intersection after the release location. This fraction then rises nearly linearly to a value of over 0.8 over the next three intersections. In the streets the downward flux comprises a larger fraction of the upward flux, starting at around 0.5 in the first street downwind of the release to over 2 over the next six streets.

Based on these observations, it is possible to identify three different regimes based on distance downwind of the source. Very close to the source, the vertical upward flux is a substantial fraction (up to around 0.25) of the horizontal flux through the network. In the intermediate region the vertical flux consists of both an upward and a downward component of comparable magnitudes, so that the net vertical flux is a smaller fraction of the horizontal flux. Further downwind, there is a qualitative difference in the behaviour in streets and intersections. In intersections the ratio of downward to upward flux approaches (but does not exceed) 1 ; hence the ratio of the net vertical flux to the horizontal flux approaches 0 . In streets the downward flux exceeds the upward flux and hence the net vertical flux becomes negative; it is a non-negligible fraction (around 0.15) of the horizontal flux. However, these differences are probably unimportant since the vertical fluxes are very small and the concentrations within the canopy and above are virtually the same at this distance. Indeed, the plume is vertically well-mixed both through the canopy and immediately above it beyond the third intersection from the source (Fig. 5). 

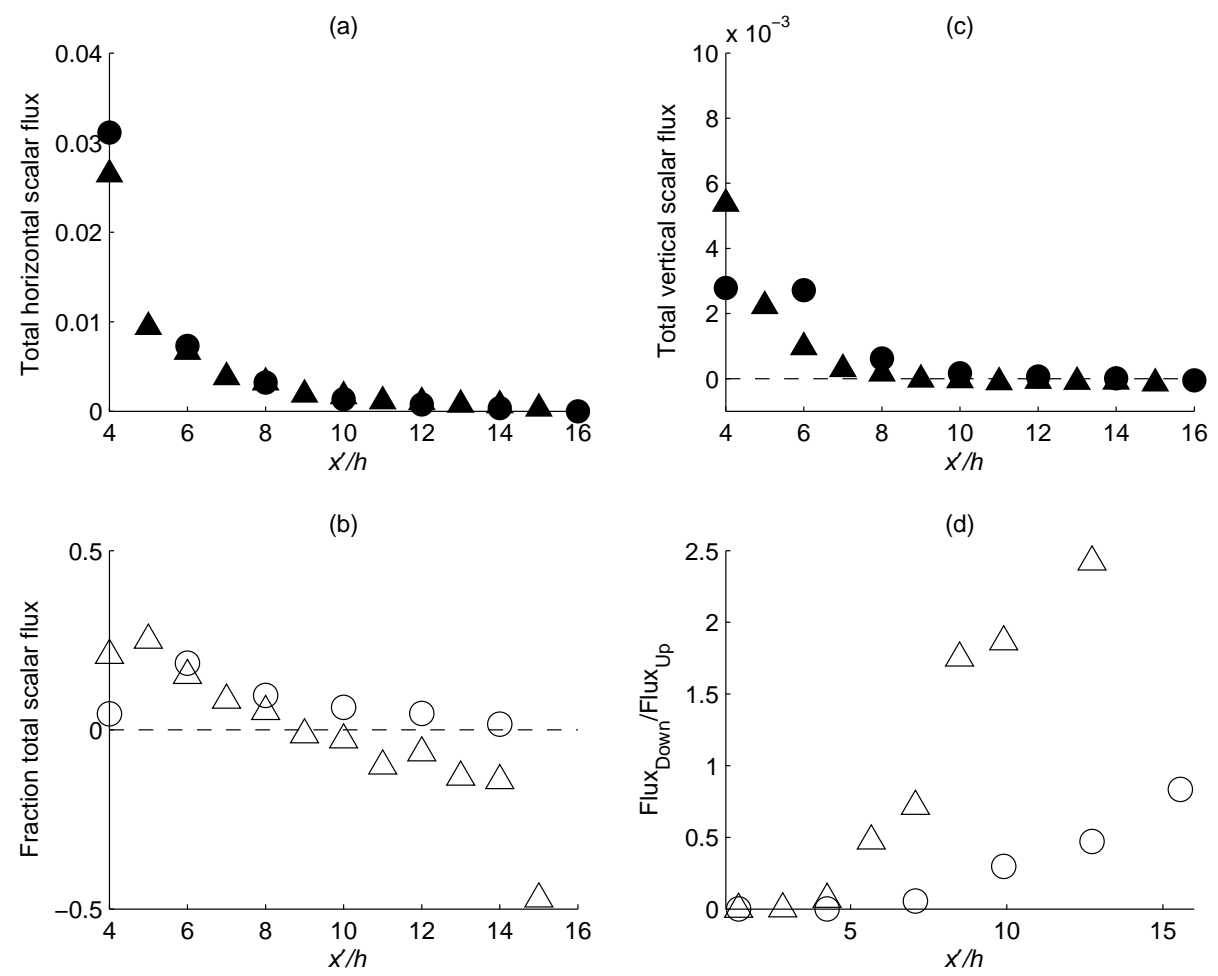

Fig. 4 Comparison between horizontal fluxes through the canopy and vertical fluxes out of the canopy top: (a) total horizontal flux, (b) total vertical flux, (c) ratio of vertical to horizontal flux, (d) ratio of downward flux to upward flux. Fluxes have been normalised using the release rate $q$.

\section{A process-based model of dispersion within and above a street} network

The results of the last section motivate an approach for modelling dispersion through a network of streets by considering the balance of fluxes through a coupled system of boxes representing each street and intersection in the network. This approach forms the basis of the SIRANE model (Soulhac, 2000; Soulhac et al., 2011, 2012), used operationally for air quality modelling. Belcher et al. (2015) recently developed an analytical model for regular street networks, which demonstrated how the geometrical and flow parameters combine into a small number of non-dimensional effective parameters that control the dispersion in the network. We now generalize the analytical model developed by Belcher et al. (2015) to include dispersion above the street network. The resulting equations cannot be solved analytically, but can be readily modelled numerically. Our aim here is to develop a minimal model that is as simple as possible while still capturing the most important processes identified from the analysis presented in Sect. 3. In doing so we do not claim that the assump- 
(a)

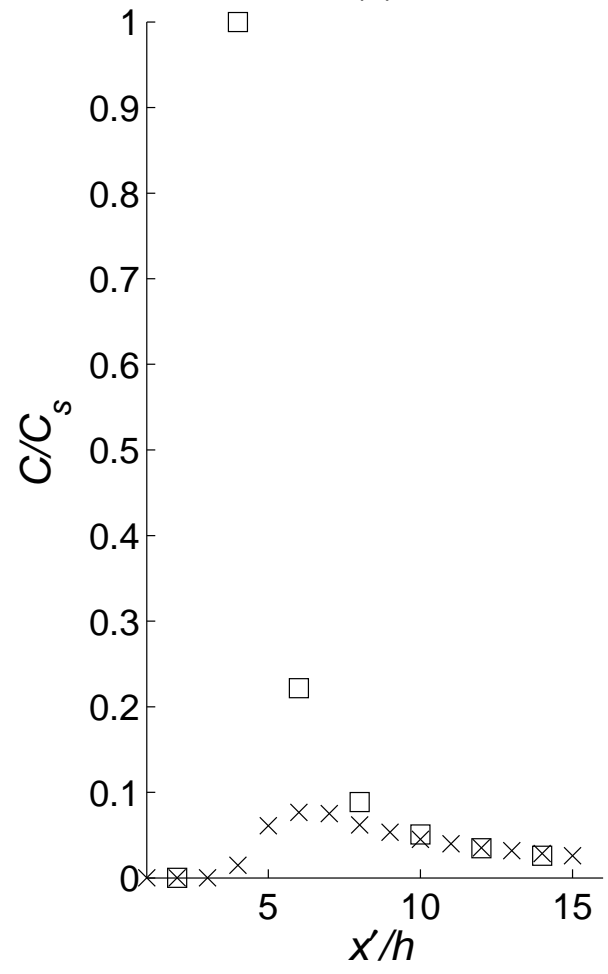

(b)

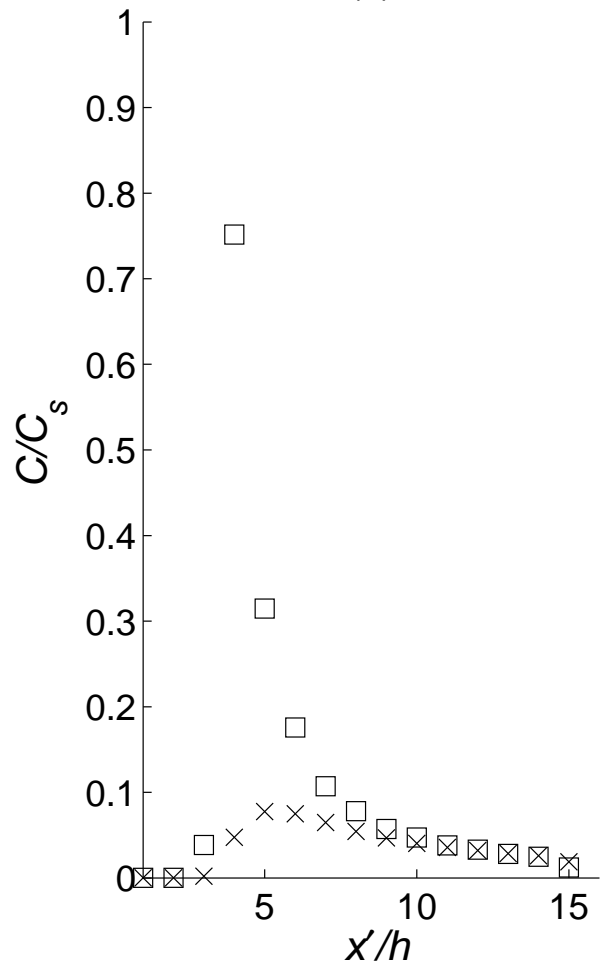

Fig. 5 Variation of mean concentration with distance from the source, (a) intersections, (b) streets. Squares: within canopy. Crosses: above canopy. The concentration is normalized by the concentration in the source box, $C_{s}$

tions made here have complete generality; indeed some of them will need to be modified in other contexts.

\subsection{Governing equations}

Following a rigorous formalism (Belcher et al., 2015), we represent each street and intersection as a box and take the volume- and ensemble-average of the scalar conservation equation over the volume $V$ of the box to give

$$
\frac{\mathrm{d} C}{\mathrm{~d} t}+\frac{1}{V} \int_{\partial V} \overline{c \mathbf{u}} \cdot \mathrm{d} \mathbf{S}=Q,
$$

where $C$ and $Q$ are the ensemble- and volume-averaged concentration and source emission rate in the box, $\partial V$ is the surface area enclosing the box, and the overline denotes an ensemble average. 
The flux term can be separated into mean and turbulent scalar fluxes,

$$
\int_{\partial V} \overline{c \mathbf{u}} \cdot \mathrm{d} \mathbf{S}=\int_{\partial V} \bar{c} \overline{\mathbf{u}} \cdot d \mathbf{S}+\int_{\partial V} \overline{c^{\prime} \mathbf{u}^{\prime}} \cdot d \mathbf{S},
$$

where primes denote fluctuations from the ensemble average. The mean and turbulent fluxes are each parametrized as described in the next section.

\subsection{Parametrization of the fluxes}

Belcher et al. (2015) show that the mean flux density $\bar{c} \overline{\mathbf{u}}$ can be written formally as the product $\langle\overline{\mathbf{u}}\rangle_{\partial V}$ of the velocity averaged over the area $\partial V$ and an average concentration $C_{a}$,

$$
\bar{c} \overline{\mathbf{u}}=C_{a}\langle\overline{\mathbf{u}}\rangle_{\partial V}
$$

In the next section, the facet-averaged mean velocity is computed from the DNS data. The formally undetermined average concentration $C_{a}$ can be approximated as the volume-average concentration in each box, assuming that the scalar is well-mixed. Goulart (2012) and Belcher et al. (2015) demonstrate that this is a reasonable approximation for the current set-up.

Following Belcher et al. (2015), the turbulent flux density is parametrized assuming the gradient diffusion model,

$$
\overline{c^{\prime} \mathbf{u}^{\prime}}=-\mathbf{K} \nabla \bar{c}
$$

where $\mathbf{K}=\operatorname{diag}\left(K_{x}, K_{y}, K_{z}\right)$ is a diagonal matrix with diagonal components equal to the eddy diffusivity coefficients $K_{x}, K_{y}$ and $K_{z}$ in the $x, y$ and $z$ directions respectively.

It is common to represent the scalar exchange between the canopy and the air above with a detrainment velocity $E$, defined as

$$
E=\frac{K_{z}}{\Delta z}
$$

where $\Delta z$ is an appropriate vertical distance, here taken to be the vertical separation between the centres of a box in the canopy and the one immediately above it.

We can generally neglect the horizontal turbulent flux within the canopy, except when the flow direction is closely aligned with one of the streets. Additionally, the mean vertical flux can be neglected in comparison with the turbulent vertical flux.

It is straightforward to discretize Eq. 3. A first-order scheme yields the following, for each box

$$
\Delta C=\frac{\Delta t}{V}\left(\sum_{k=1}^{n} F^{k}+\sum_{k=1}^{n} f^{k}+Q\right),
$$

where $F^{k}$ and $f^{k}$ are respectively the advective and diffusive scalar fluxes through each facet $k$ of the box and $n$ is the total number of facets enclosing the box. 
4.3 Calculation of model parameters from DNS

For the current DNS set-up, with the flow at $45^{\circ}$ to the regular cubical array, the horizontal facet-averaged advection velocity components $\langle\bar{u}\rangle_{k}$ and $\langle\bar{v}\rangle_{k}$ are approximately equal. Figure 6a shows the average of $\langle\bar{u}\rangle_{k}$ and $\langle\bar{v}\rangle_{k}$ computed for intersections and streets along the transects shown in Fig. 2c. The advection velocities in intersections (average value 1.13) are slightly lower than in streets (average value 1.18). The facet-averaged velocities in the boxes just above the array (around 3.4) are about three times those in the array (not shown). For comparison, Fig. 6a also shows corresponding values of 'flux velocities', defined as the ratio of $\overline{c \mathbf{u}}$ and $C_{a}$. There is a difference of around $10-15 \%$ between the facet-averaged velocities and the flux velocities. This gives an indication of the margin of error involved in using the facet velocity as an input parameter in the model.

The detrainment velocity $E$ characterizing vertical turbulent transfer out of the canopy top is computed as follows:

$$
E=\frac{\left\langle\overline{c^{\prime} w^{\prime}}\right\rangle}{\left(C_{i n}-C_{a b v}\right)}
$$

where $C_{i n}$ and $C_{a b v}$ are the box-averaged mean concentration within and above the canopy respectively, and the facet average of the vertical flux (indicated by the angled brackets) is taken over the interface separating the two boxes.

Figure $6 \mathrm{~b}$ shows the detrainment velocity at the same locations in streets and intersections as in Fig. 6a. Values are plotted only up to a distance of $8 h$ from the source since both the vertical flux and concentration difference become tiny beyond this distance, giving indeterminate values for their ratio. The difference in detrainment velocity in streets and intersections is evident. Intersections have, on average, a detrainment velocity approximately $60 \%$ larger than streets. The average detrainment velocity for streets and intersections are: $E_{s}=0.3$ and $E_{i}=0.5$.

Values for the diffusion coefficients $K_{x}, K_{y}$ and $K_{z}$ can be computed from the DNS data, with $K_{x}=K_{y}=0.5$ and $K_{z}=0.3$ used here. These values are consistent with those used in the literature for rough surfaces (e.g. Pasquill, 1962).

\section{Numerical experiments with the network model}

The parameters calculated from the DNS data in the last section are summarized in Table 1. These values are used as input to configure a set of runs with the network model described in Sect. 4.

Figure 7 shows comparisons between the mean concentrations computed by the network model (indicated by triangles) and the DNS (indicated by circles) along the plume centreline and along lateral transects at different distances from the source. The network model generally captures well both the decay 

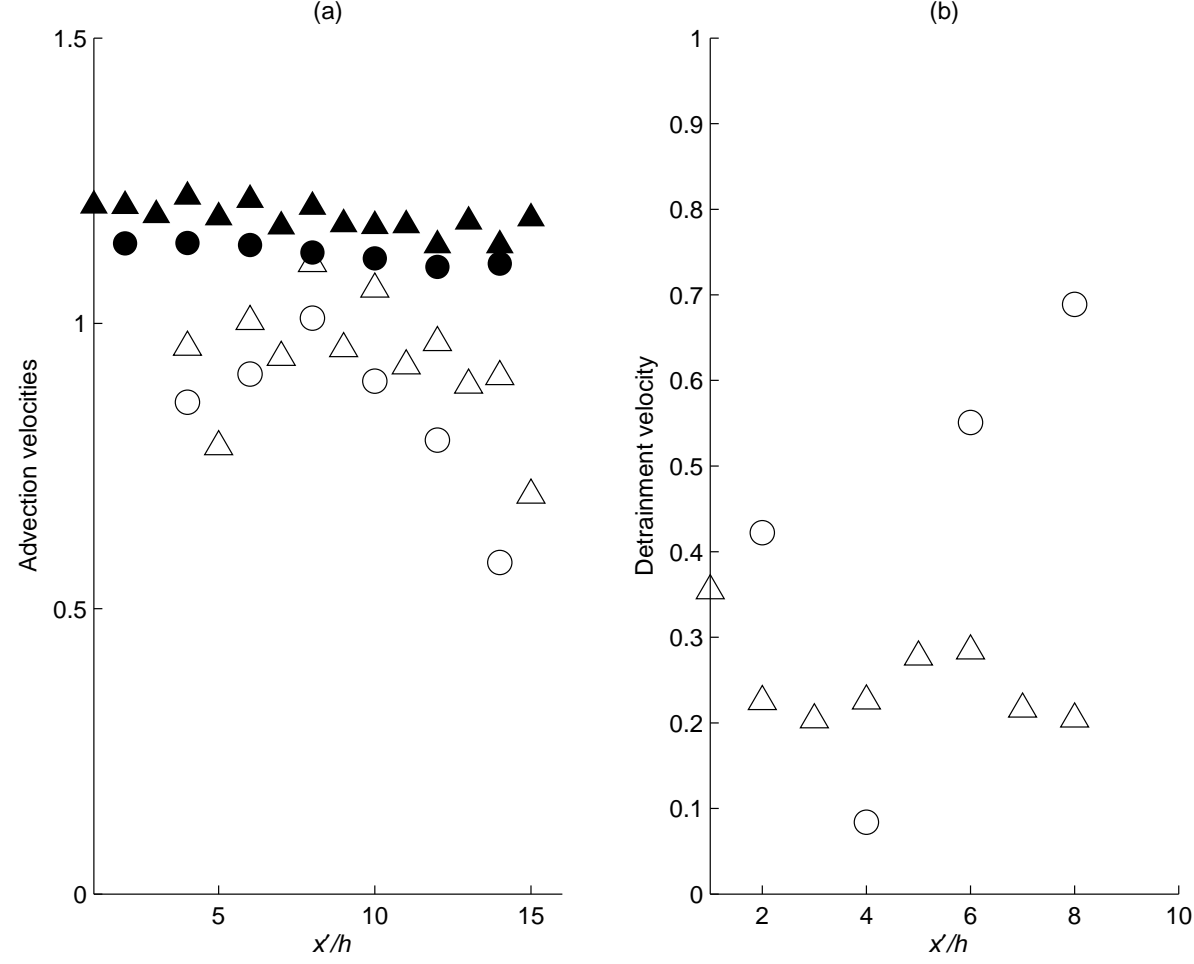

Fig. 6 (a) Filled symbols: facet-averaged advection velocities within the canopy. Empty symbols: flux advection velocity. (b) Detrainment velocities. Circles: intersections. Triangles: streets. Locations correspond to Fig. 2c.

\begin{tabular}{cccccccc}
\hline$U_{i} \approx V_{i}$ & $U_{s} \approx V_{s}$ & $E_{i}$ & $E_{s}$ & $U_{a b v} \approx V_{a b v}$ & $K_{x}$ & $K_{y}$ & $K_{z}$ \\
\hline 1.13 & 1.18 & 0.5 & 0.3 & 3.43 & 0.5 & 0.5 & 0.3
\end{tabular}

Table 1 Non-dimensional input parameters for the network model. Here $U$ and $V$ denote horizontal facet-averaged velocity components in the $x$ and $y$ directions respectively. The subscripts $i$ and $s$ refer to intersections and streets respectively, while $a b v$ refers to the layer just above the canopy layer.

in the centreline concentration and the lateral spread of the plume. The values predicted by the network model are generally within around $30 \%$ of the DNS values. This is encouraging, given the extreme simplicity of the model compared to the DNS.

Corresponding profiles in the layer just above the canopy are shown in Fig. 8. The agreement with the DNS is even better than in the canopy. It is especially good further from the source, from a distance of around $6 h \sqrt{2}$ onwards. Close to the source, at a distance of $2 h \sqrt{2}$, the model underpredicts the concentration above the canopy by up to around $30 \%$. This is consistent with an overprediction within the canopy by approximately the same amount. This is likely a result of secondary wake sources in the streets close to the 


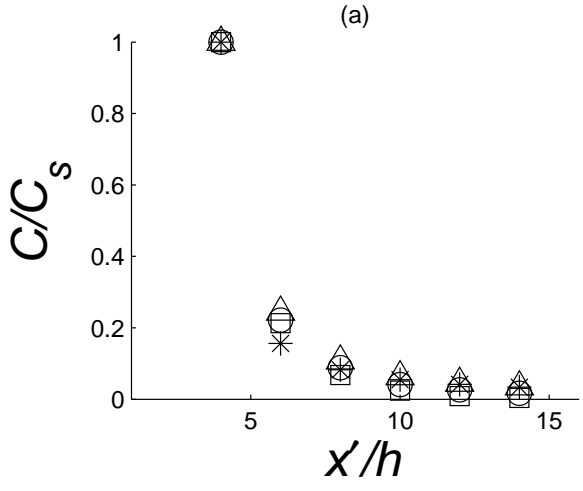

(c)

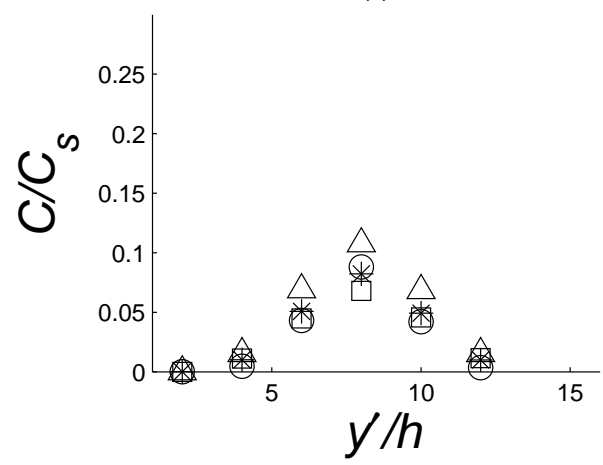

(b)

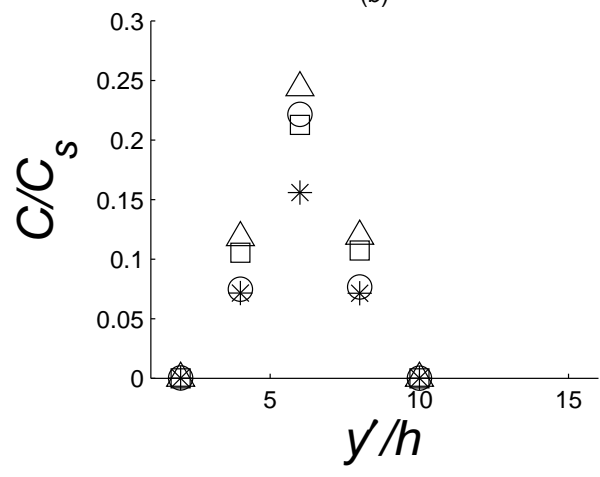

(d)

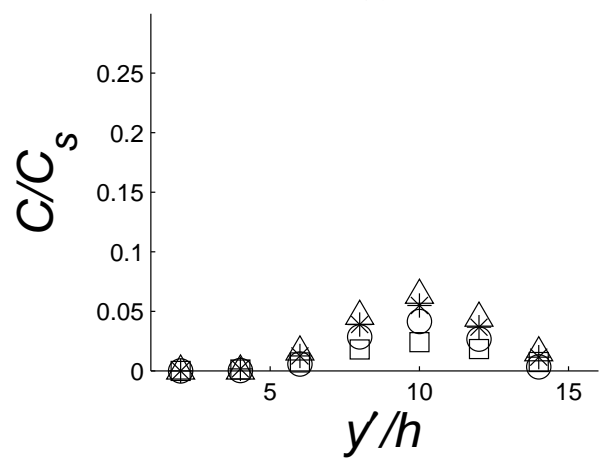

Fig. 7 Comparison between in-canopy concentration computed from network model and DNS (a) Centreline. Lateral profiles at (b) $2 h \sqrt{2}$, (c) $4 h \sqrt{2}$ and (d) $6 h \sqrt{2}$ from the source. Triangles: network model without secondary sources. Asterisks: network model with secondary sources. Squares: analytical solution of Belcher et al. (2015). Circles: DNS. Distances along and perpendicular to the plume centreline are denoted by $x /$ and $y /$ respecively.

release, which lead to an enhanced initial detrainment of material (Coceal et al. 2014). The network model does not represent these secondary sources.

A crude way to investigate the possible effect of the secondary sources is to simply increase the detrainment velocity in the relevant streets where they occur. The star symbols in Figs. 7 and 8 show the effect of increasing $E_{s}$ to 2, which is approximately 6.7 times the value in other streets. This indeed leads to closer correspondence with the DNS near the source, while the values further away are much less affected. This shows that any enhanced initial detrainment due to the secondary sources is compensated by greater re-entrainment further afield.

The sensitivity of the predicted concentrations to the input parameters is investigated by increasing and decreasing each parameter independently by $10 \%$. The concentration is then averaged along the plume centreline over six successive intersections, including the intersection in which the source is located. The averaged concentration along a lateral transect at a distance of 
(a)

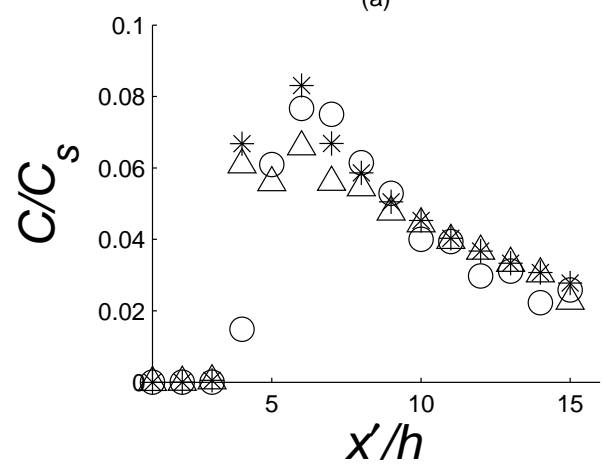

(c)

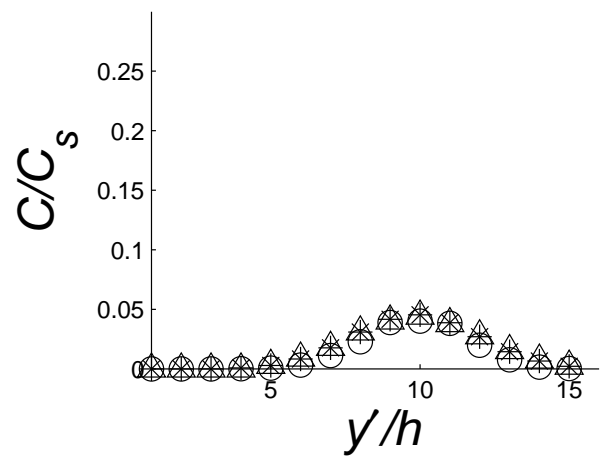

(b)

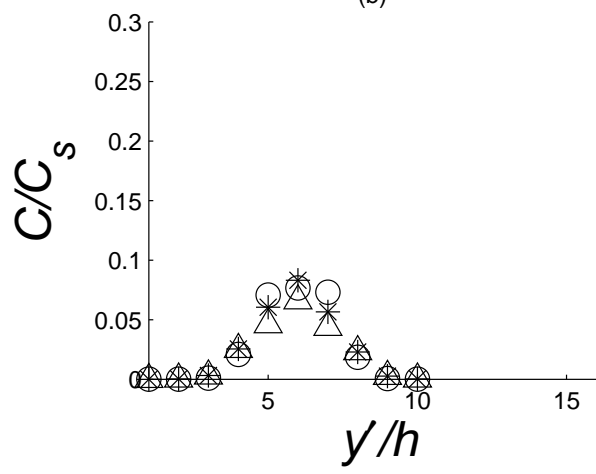

(d)

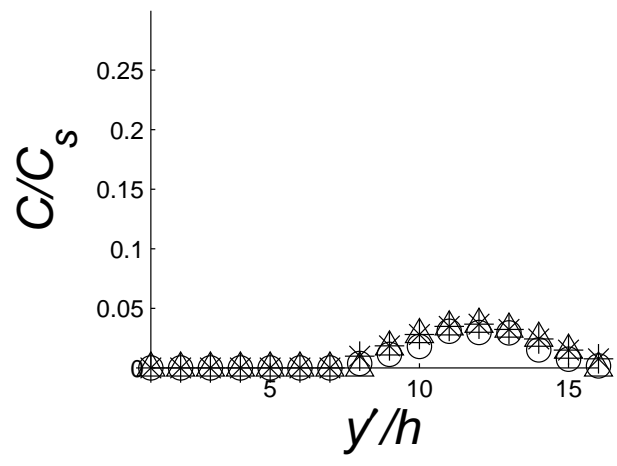

Fig. 8 Comparison between above-canopy concentration computed from network model and DNS. (a) Centreline. Lateral profiles at (b) $2 h \sqrt{2}$, (c) $6 h \sqrt{2}$ and (d) $8 h \sqrt{2}$ from the source. Triangles: network model without secondary sources. Asterisks: network model with secondary sources. Circles: DNS. $x /$ and $y /$ are the streamwise and spanwise distance to the plume centreline, resctively.

$8 h$ from the source is also computed. Similar computations are made for corresponding boxes just above the canopy layer. Table 2 shows the percentage difference in the computed concentrations relative to the run performed with the original input parameter values (as given in Table 1). The results show that changing the parameters have different effects on the concentration averaged along the centreline, and along the lateral transect. The effect on the concentration below and above the canopy are also different. On the whole the advection velocities within the canopy have the largest effect. The abovecanopy concentrations are especially sensitive to the advection velocities in the intersections, but show little dependence on the advection velocities in the streets. There is little dependence on the values of $K_{x}$ and $K_{y}$, but a change in the value of $K_{z}$ of $10 \%$ changes the concentration above the canopy by about $30 \%$ on average. 
Table 2 Network model sensitivity analysis. $D 1$ is the difference between the network model and DNS along the centerline of the plume within the canopy. $D 2$ is the difference between the network model and DNS along a lateral profile at $8 h \sqrt{2}$ from the source within the canopy. D3 is the difference between the network model and DNS along the lateral profile at $9 h \sqrt{2}$ from the source above the canopy.

\begin{tabular}{lllllll}
\hline \multirow{2}{*}{ Variables } & \multicolumn{3}{c}{ Increase of } & $10 \%$ & \multicolumn{3}{c}{ Decrease of $10 \%$} \\
& D1 & D2 & D3 & D1 & D2 & D3 \\
\hline$U_{i}, V_{i}$ & -7 & -12 & -40 & 30 & 17 & 211 \\
$U_{s}, V_{s}$ & -20 & -14 & 2 & 39 & 21 & -3 \\
$U_{a b v}, V_{a b v}$ & 13 & 10 & 71 & -11 & -9 & -33 \\
$E_{i}$ & 13 & 7 & -13 & -12 & -7 & 16 \\
$E_{s}$ & 21 & 11 & 0 & -16 & -10 & 0 \\
$K_{x}, K_{y}$ & 1 & 0 & 5 & -1 & 0 & -5 \\
$K_{z}$ & 5 & 4 & 38 & -5 & -4 & -24
\end{tabular}

\section{Conclusions}

The dispersion from a localized source within an idealized street network has been studied using DNS data. The dispersion characteristics within and above the network were compared by evaluating horizontal and vertical fluxes and their partitioning into mean and turbulent parts. The results show that the horizontal flux within the canopy is almost exclusively comprised of the mean flux, whereas above the canopy a significant counter-gradient turbulent part exists. By contrast, the vertical flux through the canopy top is generally dominated by the turbulent component. A fraction of the material originally released within the canopy and detrained into the air above is re-entrained relatively soon downstream. Based on the relative magnitude and balance of the horizontal and vertical fluxes, three distinct regions have been delineated: a near-field region, a transition region and a far-field region (summarized in simplified form in Fig. 9).

The results from the DNS have been used to develop a minimal processbased street network model that treats the dispersion within and above the network in a unified way. The model incorporates a small set of key urban dispersion processes including horizontal advection, vertical detrainment and re-entrainment. A rigorous formulation based on volume-averaging the governing equations reduced the highly complicated original problem to an effective model described by only a few parameters. Comparisons with DNS data show that this highly simplified modelling approach still gives accurate quantitative estimates of mean concentrations both within and above the street network. This indicates that the processes included in the model are indeed the most important ones and that the parametrizations on which it is based are viable. The fact that the input parameters of the simpler model were deduced from the DNS in the current exercise ensures consistency in the evaluation of the approach. Naturally, if the model were to be used in a predictive mode, it would need to be supplemented by methods to determine the parameters independently. 


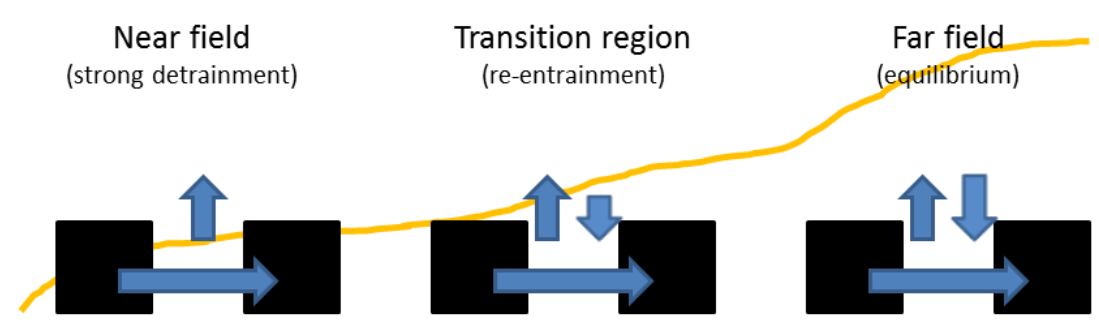

Fig. 9 Plume growth for a ground source release in an urban canopy, with arrows indicating relative magnitudes of horizontal and vertical fluxes in the near-field, the transition and the far-field regions.

The method can be readily generalized for other set-ups including nonregular geometries, although some of the specific assumptions made here may have to be modified in other scenarios. For example, the operational SIRANE model (Soulhac et al., 2011, 2012) employs a different model for parametrizing fluxes at intersections that does not assume well-mixed conditions. It also treats above-roof dispersion as a series of point sources giving rise to Gaussian plumes that are then superimposed. Moreover, as a self-contained operational model, the SIRANE model includes built-in methods for estimating the model parameters such as advection and exchange velocities. This work has focused on examining the conceptual and empirical basis of the underlying street-network approach, and to assess its performance when stripped of as many specific modelling assumptions as possible. One noteworthy result is that the basic street-network approach, as incorporated in a model much simpler than even the SIRANE model, shows a promising performance. The level of agreement obtained with the DNS data shows the predictive potential of the approach, if used in conjunction with accurate methods of estimating the model parameters. This implies that efforts to improve the SIRANE model should focus on further developing and testing such methods.

Acknowledgements Elisa V. Goulart gratefully acknowledges funding from National Council for Scientific and Technological Development (CNPq) and Espirito Santo Research Foundation (FAPES), Brazil. Omduth Coceal gratefully acknowledges funding from the Natural Environment Research Council (NERC) through their National Centre for Atmospheric Science (NCAS) under grant no. R8/H12/83/002 and from the Engineering and Physical Sciences Research Council (EPSRC contract number EP/K040707/1).

\section{References}

Belcher S (2005) Mixing and transport in urban areas. Phil Trans R Soc 363:2947-2968

Belcher S, Coceal O, Goulart E, Rudd A, Robins A (2015) Processes controlling atmospheric dispersion through city centres. J Fluid Mech 763:51-81 
Branford S, Coceal O, Thomas T, Belcher S (2011) Dispersion of a pointsource release of a passive scalar through an urban-like array for different wind directions. Boundary-Layer Meteorol 139:367-394

Britter R, Hanna S (2003) Flow and dispersion in urban areas. Annu Rev Fluid Mech 35:469-496

Carpentieri M, Robins A, Baldi S (2009) Three-dimensional mapping of air flow at an urban canyon intersection. Boundary-Layer Meteorol 133:277-296

Coceal O, Dobre A, Thomas T, Belcher S (2007) Mixing and transport in urban areas. J Fluid Mech 589:375-409

Coceal O, Goulart E, Branford S, Thomas T, Belcher S (2014) Flow structure and near-field dispersion in arrays of building-like obstacles. J Wind Eng Ind Aerodyn 125:52-68

Davidson M, Mylne K, Jones C, Phillips J, Perkins R (1995) Plume dispersion through large groups of obstacles a field investigation. Atmos Environ 29:3245-3256

Davidson M, WHSnyder, Lawson R, Hunt J (1996) Wind tunnel simulations of plume dispersion through groups of obstacles. Atmos Environ 30:3715-3725

Goulart E (2012) Flow and dispersion in urban areas. PhD thesis, University of Reading

Goulart E, Coceal O, Belcher S (2016) Spatial and temporal variability of the concentration field from localized releases in a regular building array. Boundary-Layer Meteorol 159:241-257

Hamlyn D, Hilderman T, Britter R (2007) A simple network approach to modelling dispersion among large groups of obstacle. Atmos Environ 41:58485862

Hanna S, Tehranian S, Carissimo B, Macdonald R, Lohner R (2002) Comparisons of model simulations with observations of mean flow and turbulence within simple obstacle arrays. Atmos Environ 36:5067-5079

Hilderman T, Chong R, Kiel D (2007) A laboratory study of momentum and passive scalar transport and diffusion within and above a model urban canopy. Final report Contract Report DRDC Suffield CR 2008-025

Macdonald R, Griffiths R, Cheah S (1997) Field experiments of dispersion through regular arrays of cubic structures. Atmos Environ 31:783-795

Macdonald R, Griffiths R, Hall D (1998) A comparison of results from scaled field and wind tunnel modelling of dispersion in arrays of obstacles. Atmos Environ 32:3845-3862

Milliez M, Carissimo B (2007) Numerical simulations of pollutant dispersion in an idealised urban area, for different meteorological conditions. BoundaryLayer Meteorol 122:321-342

Philips D, Rossi R, Iaccarino G (2013) Large-eddy simulation of passive scalar dispersion in an urban-like canopy. J Fluid Mech 723:404-428

Soulhac L (2000) Modelisation de la dispersion atmospheric a l'interieur de la canopee urbaine. PhD thesis, Ecole Centrale de Lyon

Soulhac L, Salizzoni P, Cierco FX, Perkins R (2011) The model sirane for atmospheric urban pollution dispersion; part i, presentation of the model. Atmos Environ 45(39):7379-7395 
Soulhac L, PSalizzoni, Mejean P, Didier D, Rios I (2012) The model sirane for atmospheric urban pollutant dispersion; part ii, validation of the model on a real case study. Atmos Environ 320-337:49

Soulhac L, Lamaison G, Cierco FX, Salem NB, Salizzoni P, Mejean P, Armand P, Patryl L (2016) Siranerisk: Modelling dispersion of steady and unsteady pollutant releases in the urban canopy. Atmos Environ 140:242-260

Vincent J (1978) Model experiments on the nature of air pollution transport near buildings. Atmos Environ 11:765-774

Yee E, Biltoft C (2004) Concentration fluctuation measurements in a plume dispersing through a regular array of obstacles. Boundary-Layer Meteorol 111:363-415

Yee E, Gailis R, Hill A, Hilderman T, Kiel D (2006) Comparison of wind tunnel and water-channel simulations of plume dispersion through a large array of obstacles with a scaled field experiment. Boundary-Layer Meteorol 121:389-432 